\title{
Caracterización de úlceras gástricas y duodenales
}

\author{
Vázquez-Anovega Héctor'1, Cruz-Carballosa Yosvanis², Cruz-Carballosa Yanelis ${ }^{3}$, \\ Calzadilla-Jardínez Iris ${ }^{4}$, Rodríguez-Zapata Rubinelda ${ }^{5}$, López-Sánchez Yamicela ${ }^{6}$
}

\section{RESUMEN}

La úlcera péptica es una lesión en la mucosa gastrointestinal (estómago o duodeno) que se extiende más allá de la muscularis mucosae y que permanece como consecuencia de la actividad de la secreción ácida del jugo gástrico. Objetivo: describir las características epidemiológicas y clínicas de las úlceras gástricas y duodenales en pacientes que acudieron al servicio de videoendoscopías del Centro Médico Alta Tecnología Hernando Dionisio Amaya Benavides de Tucupita en Venezuela, en el período 2010 - 2013. Material y métodos: se realizó un estudio descriptivo de corte transversal. Se revisaron 1972 expedientes de pacientes mayores de 15 años, procedentes de instituciones médicas de Barrio Adentro, estatales y privadas, que acudieron al servicio de videoendoscopía del Centro Médico de Alta Tecnología Hernando Dionisio Amaya Benavides de Tucupita, en el periodo 2010-2013. Se estudiaron variables demográficas y clínicas. Resultados: la morbilidad por úlceras pépticas resultó de 9,83 \%, diagnosticado en 105 pacientes, con mayor frecuencia en el sexo masculino en edades comprendidas entre 45 y 54 años. La principal procedencia en el caso de las úlceras gástricas es de los consultorios médicos populares (CMP), presente en 36 pacientes; en el caso de las duodenales, los procedentes de servicios estatales. La porción más afectada por úlceras gástricas en estómago fue el antro, con 110 úlceras diagnosticadas (94\%) y en el duodeno, cara anterior del bulbo, con 51 úlceras de las registradas en esta porción (50\%). Conclusiones: la úlcera gástrica es más frecuente que la duodenal, la epigastralgia constituyó el síntoma principal que motivó el estudio en los pacientes ulcerosos. La labor de enfermería contribuye a la realización efectiva en el servicio de videoendoscopía.

Palabras clave: gastritis, endoscopía, enfermedades duodenales. Perú. (Fuente DeCs BIREME).

\section{Characterization of gastric and duodenal ulcers}

\begin{abstract}
Peptic ulcer is an injury to the gastrointestinal mucosa (stomach and duodenum) extending beyond the muscularis mucosae and which remains as a result of the activity of acid secretion in the gastric juice. Objetive: To describe the epidemiological and clinical characteristics of gastric and duodenal ulcers in patients who attended the service videoendoscopias Medical Center of High Technology Dionisio Hernando Amaya Benavides Tucupita in Venezuela, in the 2010 period features - 2013. Material and methods: Came one descriptive cross-sectional study. 1972 records of patients older than 15 years, from Barrio Adentro medical institutions, state and private, who attended the service of High Technology videoendoscopy Dionisio Hernando Amaya Benavides Tucupita in 2010-2013 Medical Center were reviewed. Demographic and clinical variables were studied. Results: Peptic ulcer morbidity was 9.83\%, diagnosed in 105 patients, most often in men aged between 45 and 54 years. The main origin in the case of gastric ulcers is popular medical practices (CMP), present in 36 patients; in case of duodenal, those from government services. The most affected by gastric ulcers in stomach antrum portion was with 110 diahgnosticadas ulcers (94\%) and in the duodenum,

\footnotetext{
Especialista 1. ${ }^{\text {er }}$ grado en Gastroenterología. Máster en Enfermedades Infecciosas. Policlínico Universitario 26 de Julio, Mayarí. Holguín. Cuba. Máster en Nuevas Tecnologías para la Educación. Profesor asistente. Filial de Ciencias Médicas Mayarí. Holguín. Cuba.

Licenciada en Enfermería. Policlínico Universitario 26 de Julio, Mayarí. Holguín. Cuba.

Licenciada en Enfermería. Policlínico Universitario 26 de Julio, Mayarí. Holguín. Cuba.

Licenciada en Enfermería. Enfermera Endoscopista. Policlínico Universitario 26 de Julio, Mayarí. Holguín. Cuba.

Licenciada en Educación. Profesor asistente. Filial de Ciencias Médicas Mayarí. Holguín. Cuba.
} 
anterior bulb with 51 ulcers recorded in this portion (50\%). Conclusions: Gastric ulcer is more frequent than duodenal, epigastric pain was the main symptom that motivated the study ulcer patients. The nursing work contributes to the effective realization of videoendoscopy service.

Key words: Gastritis, Endoscopy, Duodenal Diseases. Peru. (Source DeCs BIREME).

\section{INTRODUCCIÓN}

La úlcera péptica es una lesión en la mucosa gastrointestinal (estómago o duodeno) que se extiende más allá de la muscularis mucosae y que permanece como consecuencia de la actividad de la secreción ácida del jugo gástrico. Desde comienzos de los años ochenta se ha producido un cambio espectacular en los conocimientos etiopatogénicos de la enfermedad ulcerosa péptica. De tal modo que hoy día las dos causas más frecuentemente relacionadas con la etiología de esta enfermedad son la infección por Helicobácter pylori y el consumo de fármacos antiinflamatorios no esteroides (AINE), incluido el ácido acetilsalicílico (AAS). Sin embargo, existen otras causas menos frecuentes que pueden producir una úlcera péptica (1).

La prevalencia actual se estima entre el 5 y el $10 \%$ de la población general (del 10 al $20 \%$ en las personas infectadas por $H$. pylori), con marcadas variaciones regionales y raciales. La incidencia anual es de aproximadamente el $1 \%$ entre las personas $H$. pylori positivas (2-5). El sexo masculino resulta más afectado que el femenino, aunque en los últimos años se ha incrementado la frecuencia de aparición de úlceras gástricas en mujeres, con la aparición de úlceras duodenales en edades más tempranas que las gástricas, con un pico alrededor de los 45 años, con localizaciones más frecuentes en la región antral para las gástricas, y el bulbo en el caso de las duodenales; el dolor es el síntoma más frecuente de la enfermedad ulcerosa péptica' (6-10).

Las úlceras pépticas constituyen afecciones frecuentes en todos los servicios gastroenterológicos a escala mundial, la mayoría de autores describen una proporción de 4:1 entre úlceras duodenales y gástricas, con predominio en el sexo masculino en relación 2:1(2). En el servicio llamó la atención el creciente número de pacientes diagnosticados con úlceras gástricas, y que sean del sexo femenino, con disminución de esa relación o proporción antes mencionada, solo comparable con Japón; ello fue el motivo para realizar este estudio, dado el desconocimiento que se tiene en nuestro estado, de la real morbilidad por estas afecciones y la magnitud del problema. Es imprescindible destacar la importancia que tiene la enfermera en la realización de estos procederes. Por lo que se propuso el objetivo de describir las características epidemiológicas y clínicas de las úlceras gástricas y duodenales en pacientes que acudieron al servicio de videoendoscopías del Centro Médico Alta Tecnología Hernando Dionisio Amaya Benavides de Tucupita en Venezuela en el período 2010 2013.

\section{MATERIAL Y MÉTODOS}

Estudio descriptivo de corte transversal. El universo fueron 1972 expedientes de todos los pacientes mayores de 15 años, a quienes se les había realizado una videoendoscopía del tracto digestivo superior en el en el Servicio de Videoendoscopía del Centro Médico de Alta Tecnología (CMDAT) Hernando Dionisio Amaya Benavides de Tucupita, Venezuela, en el período comprendido desde enero de 2010 hasta junio de 2013. Se excluyeron las embarazadas y puérperas. Se utilizó una cédula donde se consideró los siguientes datos: características demográficas (edad y sexo del paciente), lugar de residencia, institución, principales síntomas que motivaron la indicación del estudio, diagnóstico de la videoendoscopía, tipo de úlcera y su localización.

Cuando se consideró que un paciente tenía diagnóstico positivo de ulcera gástrica y duodenal se le incluyó en la muestra. La recolección la realizaron los autores, previo consentimiento informado de los pacientes. El análisis de los datos se realizó utilizando la hoja electrónica de cálculo Calc del paquete OpenOffice, se analizó la frecuencia y porcentaje por grupos de edades y sexo, su institución de procedencia, así como los principales síntomas que motivaron la indicación del estudio; se describió el tipo de úlcera y su localización, correlacionándose con posterioridad la frecuencia entre úlceras gástricas y duodenales; los resultados se expresaron en tablas.

\section{RESULTADOS}

En la tabla 1 se presenta la prevalencia general y el tipo de diagnóstico de las úlceras gastroduodenales. Se identificó que 195 pacientes (9,8 \%) fueron diagnosticados con úlceras. En relación con el tipo de úlcera diagnosticada, se identificó que 105 pacientes (5,5\%) presentaron úlce- 
ras gástricas y 90 (4,56 \%) úlceras duodenales.

En la tabla 2 se analiza la distribución por sexo de las úlceras gástricas; en este estudio predomina el sexo masculino con 54,28 \%. En el 2011 se aprecia un incremento en ambos sexos con el 16,19 \% en masculino y 18,09\% en femenino.

En la tabla 3 se puede notar que las úlceras duodenales resultaron más frecuentes en edades comprendidas entre los 45 y 54 años, siguiéndole en frecuencia las edades comprendidas entre los 25 y 34 años de edad.

En la tabla 4 se puede observar que las úlceras duodenales resultan ser más frecuentes en pacientes de sexo masculinos (59 pacientes) con 65,55 \% de los ulcerosos duodenales, casi duplicando la cifra de féminas (31 pacientes) con el 34,4\%.

En la tabla 5 se puede observar que la mayoría de pacien-

Tabla 1. Distribución de pacientes según el diagnóstico de tipo de úlceras por año en el Servicio de Videoendoscopía del Centro Médico de Alta Tecnología Hernando Dionisio Amaya Benavides de Tucupita, Venezuela.

\begin{tabular}{|c|c|c|c|c|c|c|c|}
\hline Años & $\begin{array}{l}\text { Endoscopías } \\
\text { realizadas }\end{array}$ & $\begin{array}{l}\text { Pacientes } \\
\text { con úlcera } \\
\text { gástrica }\end{array}$ & $\%$ & $\begin{array}{c}\text { Pacientes con } \\
\text { úlcera } \\
\text { duodenal }\end{array}$ & $\%$ & $\begin{array}{c}\text { Total } \\
\text { pacientes con } \\
\text { úlceras }\end{array}$ & $\%$ \\
\hline 2010 & 620 & 24 & 3,87 & 35 & 5,64 & 59 & 9,51 \\
\hline 2011 & 533 & 36 & 6,75 & 23 & 4,31 & 59 & 11,06 \\
\hline 2012 & 581 & 31 & 5,33 & 24 & 4,13 & 55 & 9,46 \\
\hline 2013 (6 meses) & 238 & 14 & 5,88 & 8 & 3,36 & 22 & 9,24 \\
\hline TOTAL & 1972 & 105 & 5,32 & 90 & 4,56 & 195 & 9,88 \\
\hline
\end{tabular}

Fuente: hoja de cargo

Tabla 2. Distribución de pacientes con úlceras gástricas según sexo en el Servicio de Videoendoscopía del Centro Médico de Alta Tecnología Hernando Dionisio Amaya Benavides de Tucupita, Venezuela.

\begin{tabular}{rrrrrrr}
\hline \multicolumn{7}{c}{ Úlceras gástricas N-105 } \\
Años & N. & \% & Masculino & \% & Femenino & \multicolumn{1}{c}{$\%$} \\
\hline 2010 & 24 & 22,85 & 16 & 15,23 & 8 & 7,61 \\
2011 & 36 & 34,28 & 17 & 16,19 & 19 & 18,09 \\
2012 & 31 & 29,52 & 15 & 14,28 & 16 & 15,23 \\
2013 & 14 & 13,33 & 9 & 8,57 & 5 & 4,76 \\
Total & 105 & 100,00 & 57 & 54,28 & 48 & 45,71 \\
\hline
\end{tabular}

Fuente: hoja de cargo

Tabla 3. Distribución úlceras duodenales en grupos etarios en el Servicio de Videoendoscopía del Centro Médico de Alta Tecnología Hernando Dionisio Amaya Benavides de Tucupita, Venezuela, en los meses de enero 2010 a junio 2013.

\begin{tabular}{|c|c|c|c|c|c|c|c|c|c|c|c|c|c|c|c|c|c|c|}
\hline \multirow{3}{*}{ Años } & \multicolumn{18}{|c|}{ Grupos edades } \\
\hline & \multicolumn{3}{|c|}{$15-24$} & \multicolumn{3}{|c|}{$25-34$} & \multicolumn{3}{|c|}{$35-44$} & \multicolumn{3}{|c|}{$45-54$} & \multicolumn{3}{|c|}{$55-64$} & \multicolumn{3}{|c|}{65 a más } \\
\hline & $\mathrm{n}$ & $\mathrm{M}$ & $\mathrm{F}$ & $\mathrm{n}$ & $\mathrm{M}$ & $\mathrm{F}$ & $\mathrm{n}$ & $\mathrm{M}$ & $\mathrm{F}$ & $\mathrm{n}$ & $\mathrm{M}$ & $\mathrm{F}$ & $\mathrm{n}$ & $\mathrm{M}$ & $\mathrm{F}$ & $\mathrm{n}$ & $\mathrm{M}$ & $\mathrm{F}$ \\
\hline 2010 & 5 & 3 & 2 & 6 & 4 & 2 & 5 & 4 & 1 & 11 & 7 & 4 & 5 & 2 & 3 & 2 & 2 & 0 \\
\hline 2011 & 2 & 1 & 1 & 6 & 6 & 0 & 6 & 3 & 3 & 3 & 2 & 1 & 4 & 3 & 1 & 3 & 3 & 0 \\
\hline 2012 & 3 & 2 & 1 & 7 & 4 & 3 & 4 & 1 & 3 & 7 & 3 & 4 & 2 & 2 & 0 & 1 & 1 & 0 \\
\hline 2013 & 2 & 1 & 1 & 2 & 2 & 0 & 1 & 0 & 1 & 1 & 1 & 0 & 1 & 1 & 0 & 1 & 1 & 0 \\
\hline Total & 12 & 7 & 5 & 21 & 16 & 5 & 16 & 8 & 8 & 22 & 13 & 9 & 12 & 8 & 4 & 7 & 7 & 0 \\
\hline
\end{tabular}

Fuente: hoja de cargo 
tes diagnosticados con úlcera gástrica procedían de CMP (36 pacientes) lo que representa el 34, 28\%; las instituciones privadas aportaron menor número de pacientes con diagnóstico de úlcera gástrica, con solo 7 casos, que representaron el 6,66 \%; no obstante lo anterior, se considera necesario establecer una serie de reflexiones en los CMP, dada la oportunidad que brinda el servicio de videoendoscopía, a fin de lograr una mayor utilización y explotación de estos servicios en el estado.
En la tabla 6 se muestra las regiones donde se localizaban las úlceras gástricas en estos pacientes; se debe aclarar que existieron pacientes con ulceras gástricas múltiples, lo cual explica el mayor número de estas (112), que pacientes (105) con ese diagnóstico; la región antropilórica resultó el lugar de afectación más frecuente, donde asentaron el 95,2 \% de las úlceras gástricas diagnosticadas, mientras que el fundus y cardias fueron los sitios donde con menos frecuencia se visualizaron las úlceras.

Tabla 4. Úlceras duodenales distribución por sexo en el Servicio de Videoendoscopía del Centro Médico de Alta Tecnología Hernando Dionisio Amaya Benavides de Tucupita, Venezuela entre los meses de enero 2010 a junio 2013.

\begin{tabular}{lcrcrrr}
\hline \multicolumn{7}{c}{ Úlceras duodenales n-90 } \\
Años & $\mathbf{n}$ & \% & Masculino & \multicolumn{1}{c}{} & Femenino & \% \\
\hline 2010 & 34 & 37,77 & 22 & 24,44 & 12 & 13,33 \\
2011 & 24 & 26,66 & 18 & 20,0 & 6 & 6,66 \\
2012 & 24 & 26,66 & 13 & 14,44 & 11 & 2,22 \\
2013 & 8 & 8,88 & 6 & 6,66 & 2 & 2,22 \\
Total & 90 & 100,00 & 59 & 65,55 & 31 & 34,44 \\
\hline
\end{tabular}

Fuente: hoja de cargo

Tabla 5. Distribución de pacientes con úlceras gástricas según procedencia en el Servicio de Videoendoscopía del Centro Médico de Alta Tecnología (CMDAT) Hernando Dionisio Amaya Benavides de Tucupita, Venezuela.

\begin{tabular}{|c|c|c|c|c|c|c|c|c|c|c|c|}
\hline \multirow{2}{*}{ Años } & \multicolumn{11}{|c|}{ Procedencia } \\
\hline & Estatal & $\%$ & Privado & $\%$ & CMP & $\%$ & CDI & $\%$ & Otros & $\%$ & Total \\
\hline 2010 & 5 & 4,76 & 0 & 0,0 & 10 & 9,52 & 4 & 3,80 & 5 & 4,76 & 24 \\
\hline 2011 & 9 & 8,57 & 5 & 4,76 & 15 & 14,28 & 2 & 1,90 & 5 & 4,76 & 36 \\
\hline 2012 & 8 & 7,61 & 1 & 0,95 & 10 & 9,52 & 4 & 3,80 & 8 & 7,61 & 31 \\
\hline 2013 & 5 & 4,76 & 1 & 0,95 & 1 & 0,95 & 1 & 0,95 & 6 & 5,71 & 14 \\
\hline Total & 27 & 25,7 & 7 & 6,66 & 36 & 34,28 & 11 & 10,47 & 24 & 22,85 & 105 \\
\hline
\end{tabular}

Fuente: hoja de cargo

Tabla 6. Distribución de pacientes con úlceras gástricas según su localización en el Servicio de Videoendoscopía del Centro Médico de Alta Tecnología (CMDAT) Hernando Dionisio Amaya Benavides de Tucupita, Venezuela

\begin{tabular}{ccccccc}
\hline \multirow{2}{*}{ Años } & \multicolumn{2}{c}{ Región antro } & \multicolumn{2}{c}{ Cuerpo gástrico } & \multicolumn{2}{c}{ Fundus y cardias } \\
& $\mathrm{N} .{ }^{\circ}$ & $\%$ & $\mathrm{~N} .^{\circ}$ & $\%$ & $\mathrm{~N} .^{\circ}$ & $\%$ \\
\hline 2010 & 23 & 21,9 & 0 & 0,00 & 0 & 0,00 \\
2011 & 31 & 29,5 & 3 & 2,56 & 1 & 0,85 \\
2012 & 31 & 29,5 & 1 & 0,85 & 1 & 0,85 \\
2013 & 15 & 14,3 & 1 & 0,85 & 0 & 0,00 \\
Total & 105 & 95,2 & 5 & 4,27 & 2 & 1,70 \\
\hline
\end{tabular}

Fuente: hoja de cargo 
Tabla 7. Síntomas en pacientes con diagnóstico de úlceras gástricas y duodenales en el Servicio de Videoendoscopía del Centro Médico de Alta Tecnología (CMDAT) Hernando Dionisio Amaya Benavides de Tucupita, Venezuela

\begin{tabular}{|c|c|c|c|c|c|c|}
\hline \multirow[t]{2}{*}{ Síntomas } & \multicolumn{2}{|c|}{$\begin{array}{c}\text { Ulceras gástricas } \\
\text { n-105 }\end{array}$} & \multicolumn{2}{|c|}{$\begin{array}{c}\text { Úlceras duodenales } \\
\text { n-90 }\end{array}$} & \multicolumn{2}{|c|}{$\begin{array}{l}\text { Total de pacientes con } \\
\text { úlceras n- } 195\end{array}$} \\
\hline & N. ${ }^{\circ}$ & $\%$ & $\mathrm{~N} .^{\circ}$ & $\%$ & N. ${ }^{\circ}$ & $\%$ \\
\hline Epigastralgia & 86 & 81,90 & 73 & 81,11 & 159 & 81,53 \\
\hline Acidez & 22 & 20,95 & 24 & 26,66 & 46 & 23,58 \\
\hline Melena & 12 & 11,42 & 14 & 15,55 & 26 & 13,33 \\
\hline Vómitos & 11 & 10,47 & 9 & 10,00 & 20 & 10,25 \\
\hline Hematemesis & 6 & 5,71 & 4 & 4,44 & 10 & 5,12 \\
\hline Dispepsia & 6 & 5,71 & 4 & 4,44 & 10 & 5,12 \\
\hline Náuseas & 7 & 6,66 & 6 & 6,66 & 13 & 6,66 \\
\hline Pérdida de peso & 2 & 1,90 & 0 & 0,00 & 2 & 1,02 \\
\hline Pirosis & 3 & 2,85 & 1 & 1,11 & 4 & 2,05 \\
\hline Palidez cutánea & 1 & 0,95 & 0 & 0,00 & 1 & 0,51 \\
\hline Halitosis & 1 & 0,95 & 0 & 0,00 & 1 & 0,51 \\
\hline Anorexia & 1 & 0,95 & 0 & 0,00 & 1 & 0,51 \\
\hline
\end{tabular}

Fuente: hoja de cargo

En la tabla 7 se recogen los principales síntomas referidos por los pacientes. Se puede notar que la epigastralgia es el síntoma más frecuente referido en todos los pacientes ulcerosos, estando presente en 159 de los 195 diagnosticados, lo cual representó el 81,53 \% de la muestra, seguido por la acidez en el 23,58 \%, la melena en el 13,33 $\%$ y los vómitos en el 10,25 \%, se observa similar comportamiento de estos síntomas tanto en los ulcerosos gástricos como duodenales.

\section{DISCUSIÓN}

La prevalencia general y el tipo de diagnóstico de la úlcera gastroduodenal han resultado similares a lo encontrado en otros estudios revisados en los que se ha planteado hasta $10 \%$ de prevalencia (1). Con relación al tipo de úlcera diagnosticada, la mayoría de la bibliografía revisada señala frecuencias de 1:4 y 1:7 entre úlceras gástricas y duodenales $(1,2)$, lo cual coincide con los resultados de este estudio y con reportes en Japón, país en el que existe una de las mayores tasas de prevalencia de úlceras gástricas y cáncer gástrico a escala mundial (1). Así mismo, con resultados encontrados por Díaz Robledo y León en un estudio realizado en Maracaibo, Venezuela, en el 2009, sobre caracterización de úlceras gastroduodenales, encontraron mayor frecuencia de úlceras gástricas que duodenales, lo cual tal vez pudiera estar relacionado con la elevada frecuencia de helicobacter pilórico en este medio, por lo que se sugiere estudios analíticos y multicéntricos para determinar este comportamiento en la población.

En este estudio predomina el sexo masculino en la distribución por sexo en las úlceras gástricas, tal como se reporta en la bibliografía consultada $(1,2,5,6)$, aunque no en la proporción esperada, pues se señala que por cada mujer con úlcera gástrica, se diagnostican dos hombres 1:2 y, en este estudio, se diagnosticaron 48 mujeres y 57 hombres con úlceras gástricas, siendo la proporción en este estudio 1:1,28, es decir, se observa una tendencia al incremento de estas úlceras en féminas como reportan algunos autores, pero más recientemente otros autores mencionan la inexistencia de diferencias en cuanto al sexo en la úlcera gástrica $(2,3)$, lo cual pudiera estar relacionado con un mayor consumo de AINEs y al incremento del tabaquismo en este sexo.

Las úlceras duodenales por grupos etarios se comportó con resultados similares a los encontrados en la mayoría de la literatura publicada, en que se señala la existencia de pico en su frecuencia a los 45 años $(3,4)$, esto pudiera estar relacionado con una mayor exposición a factores agresivos exógenos y hábitos tóxicos en esas edades, lo 
cual no constituyó objeto de análisis en esta investigación. La distribución de las úlceras duodenales por sexo se comportó como se señala en la literatura, estos resultados coinciden con todos los reportes revisados $(1,3,10)$ que plantean una mayor frecuencia de úlceras duodenales en hombres que en mujeres.

La región antropilórica es el lugar de afectación más frecuente, siendo el fundus y el cardias los sitios donde con menos frecuencia se visualizaron las úlceras, resultados similares a los descritos en la literatura (2). La epigastralgia es el síntoma más frecuente referido en todos los pacientes ulcerosos, observándose similar comportamiento de estos síntomas, tanto en los ulcerosos gástricos como duodenales, resultados similares a los reportados por diferentes autores (7-10), así como a los obtenidos por Díaz Robledo y Anely León en estudio realizado en Zulia en la República de Venezuela en el 2009.

La prevalencia de úlceras en el Servicio de Videoendoscopía fue alta. Las úlceras gástricas resultaron más frecuentes que las duodenales. Las úlceras duodenales, al igual que las gástricas, fueron más frecuentes en el sexo masculino y en edades comprendidas entre los 45 y 54 años. La localización más frecuente de las úlceras duodenales fue en cara anterior del bulbo duodenal, mientras que en las gástricas lo constituyó la región antral. La mayor procedencia de los pacientes diagnosticados con úlcera duodenal correspondió a la de los servicios estatales, y en las úlceras gástricas a los que procedían de Consultorios Médicos Populares. La epigastralgia constituyó el síntoma principal que motivó el estudio en los pacientes ulcerosos. La labor de la enfermera en los procedimientos es imprescindible para lograr una maniobra efectiva teniendo en cuenta la invasiva de este proceder.

\section{REFERENCIAS BIBLIOGRÁFICAS}

1. Díaz R, León Columbié AR. Caracterización de la úlcera gastroduodenal. Comportamiento del área de salud integral comunitaria. (Tesis). Maracaibo, Venezuela; 2009.

2. Roncali E. El sistema digestivo, ¿cómo funciona? in Trastornos Digestivos: cómo evitarlos y cómo tratarlos. Medidas Prácticas para lograr una digestión perfecta. Digital Publications, Inc.; 2010.

3. Vila Juan J., Kutz Marcos, Fernández Esparrach Gloria, López Rosés Leopoldo, Rodríguez Sarbelio, Sánchez Yague Andrés. Endoscopic submucosal dissection in Spain: outcomes and development possibilities. Rev. esp. enferm. dig. [Internet]. 2013
Oct [citado 2014 Mar 17]; 105(9): 544-552. Disponible en: http://scielo.isciii.es/scielo.php?script=sci_art text\&pid=S113001082013000900006\&lng=es.

http://dx.doi.org/10.4321/S1130-01082013000900006.

4. Blanco Avellaneda Camilo, Aponte Martín Diego, Forero Acosta Alix Yineth, Flores Nadia Sofía, Cañadas Raúl, Peñaloza Ramírez Arecio et al. Primer consenso colombiano sobre la práctica de endoscopia digestiva «Acuerdo en lo fundamental»: Segunda parte. Aspectos éticos. Rev Col Gastroenterol [Internet]. 2013 [citado 2 abr 2014]; 28 (1): 27-44. Disponible en: http://www.scielo.org.co/pdf/rcg/v28n1/ v28n1a05.pdf

5. Le TH, Fantry GT. Peptic Ulcer Disease. Hepatol. 2005; 3 (9): 859-64.

6. González Carbajal Pascual MG, Sevilla Mederos LF, Grá Oramas B. Alteraciones histológicas de la mucosa gástrica y prevalencia del Helicobacter pylori en pacientes dispépticos. Rev Panam Infectol. 2005; 7 (1): 8-15.

7. Montes Teves P, Salazar Ventura S, Monge Salgado E. Características de las úlceras gastroduodenales en pacientes con biopsia negativa para Helicobacter pylori. Acta Gastroenterológica Latinoamericana. 2010; 140 (1): 40-45.

8. Schmidt H, Andres S, Kaakoush N, Engstrand L, Eriksson L, Goh K, et al. The prevalence of the duodenal ulcer promoting gene (dupA) in Helicobacter pylori isolates varies by ethnic group and is not universally associated with disease development: a case-control study. Gut Pathog. 2009; $1: 5$.

9. López T, M Perrone, Correnti M, Ortiz D, Cavazza ME, Avila M. Evaluación de la respuesta de inmunoglobulina a secretora anti-helicobacter pylori en saliva de pacientes con gastritis crónica. Acta odontol. venez [Internet]. 2008 [citado 19 jun 2012]; 46 (4): 440-445. Disponible en: http:// www.scielo.org.ve/pdf/aov/v46n4/art07.pdf

10. González López Lidice, Rodríguez González Boris Luis. Patogénesis de la infección por Helicobacter pylori. Rev cubana med [Internet]. 2011 Dic [citado el 23 de abril 2013]; 50 (4): 441-452. Disponible en: http://scielo.sld.cu/scielo.php?script=sci_art text\&pid=S003475232011000400010\&lng=es.

11. Zeng J, Liu JS. Quality of life after three kinds of esophagectomy for cancer. World J Gastroenterol 2012; 18: 5106-5113.

12. Yamazato T, Oyama T, Yoshida T, Baba Y, Yamanouchi K, Ishii Y, et al. Two years' intensive training in endoscopic diagnosis facilitates detection of early gastric cancer. Intern Med 2012;51:1461-1465 
13. Hernández Conde R, Noa Pedroso G, Domínguez Álvarez C, Mora Díaz I, Osorio-Pagola M, Pomares Pérez Y. Caracterización de pacientes con úlceras pépticas negativas a Helicobacter pylori. Medisur [Internet]. 2013 [citado 23 abr 2014]; 11(5): 13. Disponible en: http://www.medisur.sld.cu/index.php/ medisur/article/view/2146

\section{Correspondencia}

Yosvanis Cruz Carballosa

Gonzalitos 1500 Norte, Monterrey, Nuevo León. México.

Telefóno: 83481010, 0448113902312

Correo electrónico: yosvanis@mayari.hlg.sld.cu

Forma de citar este artículo: Vázquez-Anovega H, CruzCarballosa Y, Cruz-Carballosa Y, Calzadilla-Jardínez I, RodríguezZapata R, López-Sánchez Y. Caracterización de úlceras gástricas y duodenales, Servicio de Videoendoscopía del centro médico de alta tecnología (CMDAT) Hernando Dionisio Amaya Benavides de Tucupita - Venezuela -2013. Rev. enferm Herediana.2014;7(1):3-9.

Fecha de recepción: 23 abril de 2014

Fecha de aceptación: 12 de mayo de 2014 